Document downloaded from:

http://hdl.handle.net/10251/81464

This paper must be cited as:

Girbés, V.; Armesto Angel, L.; Dols Ruiz, JF.; Tornero Montserrat, J. (2017). An Active Safety System for Low-Speed Bus Braking Assistance. IEEE Transactions on Intelligent Transportation Systems. 18(2):377-387. doi:10.1109/TITS.2016.2573921.

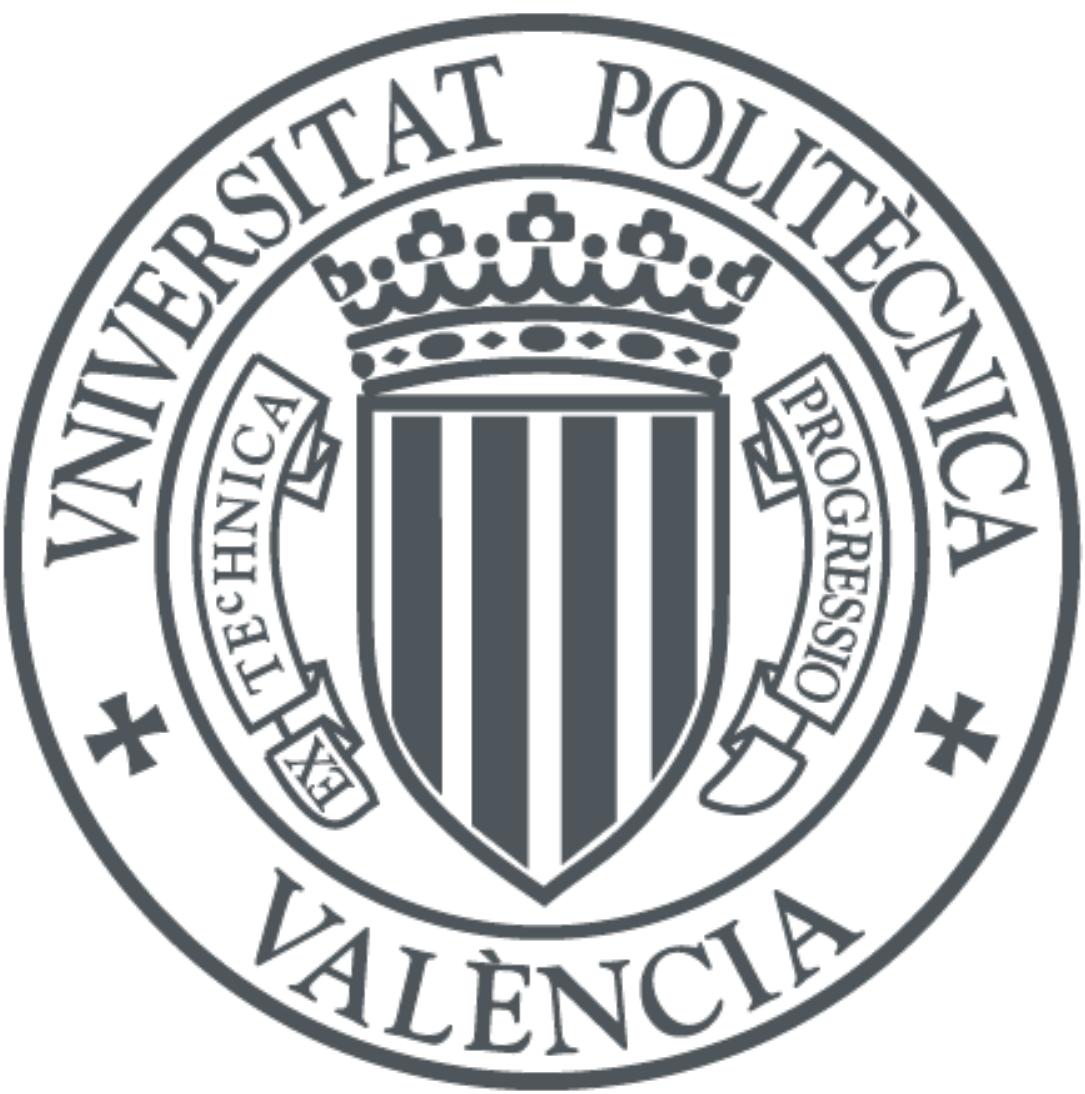

The final publication is available at

Copyright Institute of Electrical and Electronics Engineers (IEEE)

Additional Information 


\title{
An Active Safety System for Low-Speed Bus Braking Assistance
}

\author{
Vicent Girbés, Leopoldo Armesto, Juan Dols and Josep Tornero
}

\begin{abstract}
Accidents in which buses or coaches are involved cause thousands of injuries and fatalities every year. To reduce their number and severity, the paper describes an Advanced Driver Assistance Systems (ADAS) based on a haptic throttle pedal and emergency braking. It also proposes a computationally efficient algorithm with a methodology based on three main concepts: a simplified but accurate vehicle model; an efficient collision detection system considering driver's intention and pedestrians wandering around the vehicle; and a risk evaluation system to generate warnings and emergency braking signals. Finally, the performance of the proposed ADAS is validated using a driving simulation cabin with a very realistic urban scenario and original elements from real buses. The results show a statistically significant improvement in safety, as the number of collisions and high risk situations are clearly minimized, reaction time to press the brake pedal is improved and time to collision increased in emergency situations. Implementation of the proposed ADAS into city buses would potentially improve safety, reducing the frequency and severity of accidents with pedestrians.
\end{abstract}

\section{INTRODUCTION}

The World Health Organization, in its global status report on road safety 2015 [1], states the need for governments to ensure that public passenger transportation systems (PTS) are safe, affordable, accessible and sustainable, as this will lead to a reduction in the number of traffic accidents and fatalities.

It is a fact that buses and coaches are essential components of PTS and have socio-economic impact on the promotion of tourism, energy saving, environmental protection, road safety, mobility and economy. Indeed, no other mean of collective passenger transport plays such an important role in all these areas at the same time. In particular, buses represent $55 \%$ of land PTS in the European Union (EU), while other PTS - rail, tram and metro - together constitute the remaining 45\% [2].

Although bus accident figures are significantly lower than other means of transport, the severity of their accidents and the fact that they are massive PTS imply a continuous search for solutions to improve active and passive safety. This is being one of the main fields of technological development within the sector and an absolute priority for all PTS operators. In this sense, in the last European Commission Framework Programs and $\mathrm{H} 2020$, there have been several projects to classify, analyse and prevent accidents (TRACE, SafetyNet, DACOTA, LIVE), while others were intended to design passive and active safety systems (ADVISORS, HASTE, WATCH-OVER).

From 2004 to 2013 buses and coaches represented around $3 \%$ of registered traffic fatalities in the EU [3]. In 2013 about $50 \%$ of fatalities in bus or coach accidents occurred in urban areas. Moreover, almost $30 \%$ of those who died in 2013 in road accidents that involved buses or coaches were pedestrians and more than $20 \%$ were vehicle occupants [3]. In Spain, in

V. Girbés, L. Armesto, J. Dols and J. Tornero are with Instituto de Diseño y Fabricación at Universitat Politècnica de València, Spain
2013 there were 1816 accidents with victims (mortal or with injuries) in which a bus was involved [4], where $85 \%$ of those accidents happened in urban areas.

An analysis from police and hospital data regarding safety in buses (including transfers to/from bus stops) was presented in [5]. The authors concluded that most injuries occurred on board, as a result of an abrupt change in vehicle speed $(60 \%$ in braking and $25 \%$ in acceleration), mainly affecting elderly and disabled people. This group is shown as the most vulnerable because it represents more than $50 \%$ of accidents, both as passengers (greater harmfulness when getting off the bus than when getting on it) and as pedestrians (around bus stops).

Indeed, one of the most common difficulties for drivers to avoid accidents involving pedestrians is their lack of visibility, particularly accidents involving children and wheelchairs. For instance, in the USA from 2003 to 2012, 119 school-age children died in school-transportation-related crashes while they were moving around the vehicle [6]. Moreover, most accidents (crashes and fatalities) happened due to impacts in the front and right side of the bus.

From CARE database [3], it can be observed that in accidents where a pedestrian was involved around $22 \%$ were caused by a late driver action, while in more than $32 \%$ there was no action at all. Most of those accidents could have been avoided by a warning system or a collision avoidance system with emergency braking. Indeed, a study carried out in the EC FP6 "Project Cost-benefit assessment and prioritisation of vehicle safety technologies", the authors concluded that front and side collision warning systems can reduce an $8 \%$ the severity and a $12 \%$ the risk of collisions of type rear-end and head-on collisions, as well as vehicle-pedestrian collisions. On the other hand, Intelligent Speed Adaptation (ISA) systems could reduce around a $50 \%$ the risk of collision on these types of accidents.

Therefore, active safety systems covering the periphery of the bus become necessary because mirrors are insufficient since the location of a person can change quickly and the driver may be distracted. In this sense, systems to support blind spots check to warn the driver of pedestrians or objects near the vehicle have been developed during the last decade [7][9]. Once an obstacle has been detected, Advanced Driver Assistance Systems (ADAS) normally produce audiovisual or haptic warnings [10]-[15], with the aim of reducing the number of accidents and their severity.

In [16], it was stated that the most effective active safety systems to avoid frontal and lateral crashes are those based on rear-end, head-on and side collision warning systems. So, in order to improve traffic safety, these systems try to modify driver's behaviour through reactive stimuli to influence their decision making, for instance by avoiding a rear-end or headon accident [17]-[19], helping in lane changing [20] or in lane keeping manoeuvres [21]. 
At present, there are already systems using a gas pedal for active car-following with speed adaptation [22]-[24]. Many other studies focus on the development of methods to evaluate and verify the quality, safety and functionality of these systems, and the analysis of the effects they cause in relation to security when they are included in the vehicle control chain [25], [26]. On the other hand, other researchers try to analyse the adaptation of the driver to the new device, demonstrating that driving can be improved after a certain time of usage, making it safer [27]-[29]. See [30] for a detailed survey on the effect of haptic support systems on driver's performance.

The main contribution of this paper is the development of a new algorithm to generate warnings and emergency braking signals in ADAS, particularly suited to low-speed manoeuvres in urban environments, mostly in areas of passenger loading and unloading. In particular, we propose a computationally efficient algorithm with a methodology based on three main concepts: a simplified vehicle model; an efficient collision detection system based on the arc reachable manifold, considering driver's intention and pedestrians moving around the vehicle; and a risk evaluation system to generate warnings and emergency braking signals. A driving simulator study was conducted using a realistic urban environment, which shows the advantages of the proposed active safety system in reducing the number of collisions and high risk incidents. Driver's reaction time is also improved and as a consequence the time to collision is increased.

The proposed safety system is composed by a pedestrian detection module based on smart cameras placed in the periphery of the bus, a haptic feedback device in the throttle pedal and an emergency braking system. However, we assume that pedestrians are previously detected and this information is known, therefore pedestrian detection is out of the scope of this paper. To the best of authors' knowledge, there is no system working on public PTS to avoid collisions with pedestrians at low speed. In this sense, the implantation of such safety system into city buses seeks to reduce the frequency and severity of accidents and injuries involving buses.

The paper is organised as follows: In Section II the proposed methodology for advanced driving systems is described. Section III describes the experimentation setup implemented to validate the performance of the ADAS system. Section IV shows the results obtained from the performed tests. Finally, a discussion is carried out in Section $\mathrm{V}$ and some conclusions are drawn in Section VI. Compliance with current legislation and relevant approvals are out of the scope of this paper.

\section{Proposed Methodology For Driver Assistance}

The methodology presented in this paper considers not only the vehicle behaviour but also pedestrians moving around it. For that purpose, it is necessary to know driver's intention, which is reduced to know the position of throttle and brake pedals, as well as the steering wheel angle. In addition to this, sensorization is required to provide information about each pedestrian detected in the vehicle surroundings: position, speed and direction of movement relative to the vehicle. As explained in Sections II-D and II-E, this is accomplished by a set of smart cameras and electro-mechanical components.

\section{A. Vehicle Model}

Let's assume a vehicle moving on a $\Re^{2}$ workspace $\mathcal{W}$ and configuration space $\mathcal{C S}$ in $\Re^{2} \times \mathcal{S}^{1}$. Where, the configuration space vector $\mathbf{q}(t)=[x(t), y(t), \theta(t)]$ indicates the Cartesian position and orientation of the vehicle. In addition to this, let $\mathcal{U}$ be the driver's space with $\mathbf{u}(t)=\left[u_{a}(t), u_{b}(t), \alpha(t)\right]$ as input vector containing the throttle pedal position $u_{a}(t)$, the brake pedal position $u_{b}(t)$ and the steering wheel angle $\alpha(t) \in$ $\left[-\alpha_{\max }, \alpha_{\max }\right]$, with $\alpha_{\max }$ as the maximum turning angle. For simplicity, pedals ranges are normalized, so that $u_{a}(t) \in[0,1]$ and $u_{b}(t) \in[0,1]$.

Our methodology is inspired by [31], [32], where non-linear longitudinal dynamics of a vehicle are based on physical laws. We decompose the components of the vehicle velocity $v(t) \in$ $\left[0, v_{\max }\right]$ (with $v_{\max }$ as the maximum linear velocity) into two velocities, one related to acceleration $v_{a}(t)$ and another related to braking $v_{b}(t)$ :

$$
v(t)=v_{a}(t)+v_{b}(t)
$$

The active safety system is designed to work at low speed, as explained in Section II-C. In this sense, most of the nonlinearities can be neglected and the non-linear model for the acceleration component is approximated by a linearised firstorder system with a delay in order to take into account the transmission dynamics.

$$
\frac{v_{a}(s)}{u_{a}(s)}=\frac{K \cdot e^{-\tau_{d} s}}{\tau s+1}, \frac{d(s)}{v_{a}(s)}=\frac{1}{s}
$$

where $d(s) \equiv \mathcal{L}[d(t)]$ is the travelled distance, $v_{a}(s) \equiv$ $\mathcal{L}\left[v_{a}(t)\right]$ and $u_{a}(s) \equiv \mathcal{L}\left[u_{a}(t)\right]$, being $\mathcal{L}$ the Laplace transform.

In order to validate the performance of the simplified model, we have obtained velocity data from a real urban bus (TEMPUS bus manufactured by Castrosua S.A.), accelerating at full throttle during 15 seconds. The fitting performance of the simplified model to the data is $R^{2}=0.973$ and the mean squared error $M S E=0.015 \mathrm{~m} / \mathrm{s}$, while the best fitting we could find using longitudinal dynamics described in [31] and Pacejka's tire model [32] is $R^{2}=0.976$ with $M S E=0.012$ $\mathrm{m} / \mathrm{s}$. In addition to this, to simulate 15 seconds using a MatlabSimulink implementation with ODE45 solver and variable simulation step, the computation time is $t_{\text {proc }}=0.153 \mathrm{~s}$ on average for the simplified first-order model. Whilst for the complex model it is $t_{\text {proc }}=13.191 \mathrm{~s}$, around 86 times slower. The computer used in the simulation is based on Ubuntu 14.04 and has the following technical specifications: CPU Intel Core i7-2670QM 2.20GHz, with 8GB DDR3 $1333 \mathrm{MHz}$.

On the other hand, the model relating the effective braking speed $v_{b}(s) \equiv \mathcal{L}\left[v_{b}(t)\right]$ with the deceleration $a_{b}(s) \equiv \mathcal{L}\left[a_{b}(t)\right]$ is described as an integrator:

$$
\frac{v_{b}(s)}{a_{b}(s)}=\frac{1}{s}
$$

The braking deceleration $a_{b}(t)$ mainly depends on brake pedal position $u_{b}(t)$, but also on the current vehicle velocity $v(t)$, as shown in Figure 1. After some tests, we detected a saturation of the braking force for values of $u_{b}(t)$ close to the maximum $u_{b}(t)>0.8$, which makes the relation between the 


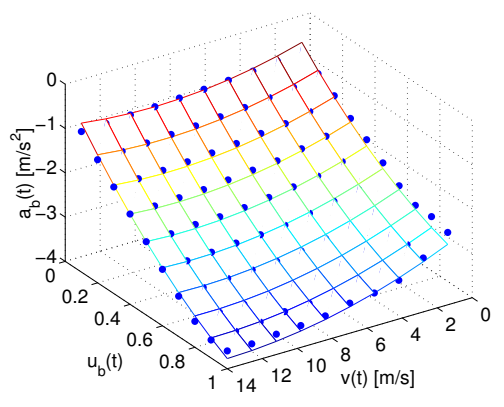

Figure 1. Deceleration surface of the second order polinomial model. Blue dots are the values of $a_{b}(t)$ measured during the braking calibration tests. $u_{b}(t)$ and $v(t)$ are normalized brake position and vehicle speed, respectively, which are used to approximate the value of $a_{b}(t)$ using Equation 4.

overall braking deceleration ${ }^{1}$ and brake pedal position slightly non-linear. We have determined that a quadratic model as in (4) is able to provide a good fitting performance. Using the Least Squares method and real data, we obtained a fitting of $R^{2}=0.988$ with $M S E=0.001 \mathrm{~m} / \mathrm{s}^{2}$ between these variables, although other approximations might be valid as well, for instance using neural networks.

$$
a_{b}(t) \approx k_{0}+k_{1} v(t)+k_{2} u_{b}(t)+k_{3} v^{2}(t)+k_{4} u_{b}^{2}(t)+k_{5} v(t) u_{b}(t)
$$

It is interesting to remark that using the same Pacejka's complex model for the braking deceleration [32], we performed parameters identification in order to adjust the model as close to real data as possible. The best fitting performance that we got was $R^{2}=0.926$ and $M S E=0.065 \mathrm{~m} / \mathrm{s}^{2}$. We found that this dynamic model differs from the real behaviour of the vehicle mostly at low speeds and with low values of braking torque, which makes more difficult to find an appropriate parameter fitting. As a conclusion, our approach is more efficient computationally because the braking value is obtained directly from a polynomial equation, without the need to compute the dynamic equations of the complex model.

\section{B. Collision Detection}

An Arc Reachable Manifold [33] is a two-dimensional manifold in $\Re^{2} \times \mathcal{S}^{1}$. Let $A R M_{v}$ be the driver's configuration space (blue arrow in Figure 2), obtained from a nonholonomic standard car-like kinematic model (i.e. type $(1,1)$ in [34], which is kinematically equivalent to Ackermann steering model). Without loss of generality, $A R M_{v}$ has its center at $\left(x_{v}, y_{v}\right)$ with $x_{v}=0, y_{v}=\operatorname{sign}(\alpha(t)) \cdot R_{v}(t)$ and radius $R_{v}(t)=\left|\frac{L}{\tan (\alpha(t))}\right|$, being $\alpha(t)$ the vehicle steering wheel angle and $L$ the wheelbase, i.e. the distance between vehicle's front and rear wheels axles.

Now, let's define $l$ as the distance from the rear axle to the front of the vehicle and $w$ as the vehicle width assuming a rectangular footprint. In order to abstract pedestrians' shape, vehicle footprint is usually inflated with the estimated radius of the circle circumscribing a pedestrian $R_{p}$ (see Figure 2). Thus, following the ideas of [35], we can obtain a parametrized rect-

\footnotetext{
${ }^{1}$ Measured as the ratio between the time necessary to stop the vehicle and the initial velocity when the pedal is pressed.
}

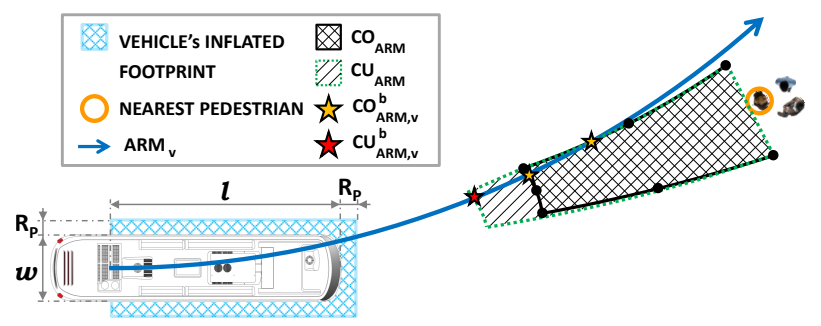

Figure 2. Example of collision detection, where $C O_{A R M}$ and $C U_{A R M}$ spaces are computed from nearest pedestrian position using vehicle's inflated footprint, while collision points $C O_{A R M, v}^{b}$ and $C U_{A R M, v}^{b}$ are computed taking into account the manifold $A R M_{v}$ from driver's inputs.

angular shaped vehicle (chequered cyan rectangle in Figure 2):

$$
\begin{aligned}
& x_{v}(\lambda)=\left\{\begin{array}{cc}
d Y \tan \lambda & \text { if } \tan \lambda<\frac{d X}{d Y} \text { and } \lambda<\frac{\pi}{2} \\
-d Y \tan \lambda \text { if } \tan \lambda>-\frac{d X}{d Y} \text { and } \lambda>\frac{\pi}{2} & \text { otherwise } \\
d X & \text { if } \tan \lambda<\frac{d X}{d Y} \text { and } \lambda<\frac{\pi}{2} \\
-d Y & \text { if } \tan \lambda>-\frac{d X}{X Y} \text { and } \lambda>\frac{\pi}{2} \\
d Y & \text { if } \tan \lambda \geq \frac{d X}{d Y} \text { and } \lambda<\frac{\pi}{2}
\end{array}\right. \\
& y_{v}(\lambda)=\left\{\begin{array}{cc}
\frac{d}{2} \\
-d X \tan \left(\frac{\pi}{2}-\lambda\right) \\
d X \tan \left(\frac{\lambda-\pi}{2}\right) & \text { if } \tan \left(\lambda-\frac{\pi}{2}\right)<-\frac{d Y}{d X} \text { and } \lambda \geq \frac{\pi}{2}
\end{array}\right.
\end{aligned}
$$

with $\lambda \in[0, \pi]$, being $d X=l+R_{p}$ and $d Y=w / 2+$ $R_{p}$. For simplicity, we only consider the front part of the bus shape because the vehicle is moving forward. However, rear part would be treated in a similar way when moving backward.

There exists a configuration obstacle $A R M\left(C O_{A R M}\right)$ that defines the region at which the vehicle collides with objects by describing an arc (chequered black region in Figure 2). Indeed, the configuration obstacle for all pedestrians is $C O_{A R M}=$ $\cup_{i} C O_{A R M, i}$, where $C O_{A R M, i}$ denotes the configuration obstacle for the $i$-th pedestrian. From now on, we remove subindex $i$ to keep notation clearer, restricting ourselves to one single pedestrian case, although computations must be carried out for each pedestrian.

Let's denote $\left\{x_{C O_{A R M}}^{b}, y_{C O_{A R M}}^{b}\right\} \in C O_{A R M}^{b}$ the boundary points for a given pedestrian position $\left\{x_{p}, y_{p}\right\}$, being $C O_{A R M}^{b}$ the boundary of $C O_{A R M}$. This boundary can be analytically computed for any shape [35]:

$$
\begin{aligned}
& x_{C O_{A R M}}^{b}(\lambda)=c \cdot\left(x_{p}+x_{v}(\lambda)\right) \\
& y_{C O_{A R M}}^{b}(\lambda)=c \cdot\left(y_{p}-y_{v}(\lambda)\right)
\end{aligned}
$$

with $c=\frac{\left[y_{p}^{2}-y_{v}^{2}(\lambda)+x_{p}^{2}-x_{v}^{2}(\lambda)\right]\left[\left(y_{p}-y_{v}(\lambda)\right)^{2}+\left(x_{p}-x_{v}(\lambda)\right)^{2}\right]}{\left(y_{p}-y_{v}(\lambda)\right)^{4}+2\left(x_{p}^{2}+x_{v}^{2}(\lambda)\right)\left(y_{p}-y_{v}(\lambda)\right)^{2}+\left(x_{p}^{2}-x_{v}^{2}(\lambda)\right)^{2}}$.

Instead of considering the whole parametrized $C O_{A R M}^{b}$ for any arbitrary shape, only mid and end points of each segment of the rectangular shape are considered. This reduces the computation of shape abstraction to a total of 7 points (black dots in Figure 2) corresponding to $\lambda_{0}=0$, $\lambda_{1}=\arctan (d X / 2 d Y), \lambda_{2}=\arctan (d X / d Y), \lambda_{3}=\pi / 2$, $\lambda_{4}=\pi / 2+\arctan (d Y / d X), \lambda_{5}=\pi-\arctan (d X / 2 d Y)$ and $\lambda_{6}=\pi$. Furthermore, we approximate an arc segment for every triple of points related to each segment of the rectangle, having a total of 3 arcs defining an approximation of $C O_{A R M}^{b}$ :

$$
C O_{A R M}^{b} \approx C O_{A R M, L} \cup C O_{A R M, F} \cup C O_{A R M, R}
$$

where $C O_{A R M, L}$ denotes configuration obstacle $A R M$ for leftside (using points with $\lambda_{0}$ to $\lambda_{2}$ ), $C O_{A R M, F}$ corresponds to 


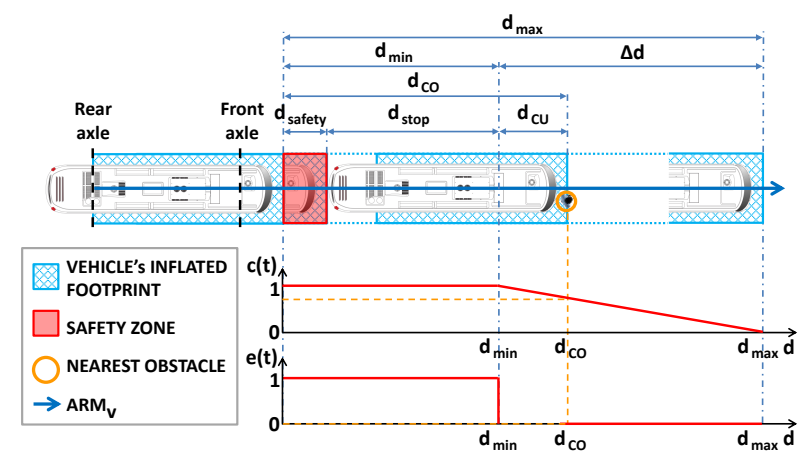

Figure 3. Collision risk evaluation and involved variables. The example is particularized for a pedestrian distance $d_{C O}$, although it is possible to analyse $c(t)$ and $e(t)$ by moving the pedestrian along the distance axis $d$.

the front-side (using points with $\lambda_{2}$ to $\lambda_{4}$ ) and $C O_{A R M, R}$ to the right-side (using points with $\lambda_{4}$ to $\lambda_{6}$ ). As a consequence, $C O_{A R M}^{b}$ becomes a piece-wise function formed by 3 arc segments, which are easier to deal with than computing the analytical locci as in (7)-(8).

Now, let's define $C O_{A R M, v}^{b}=C O_{A R M}^{b} \cap A R M_{v}$ as the manifold in $\Re^{2} \times \mathcal{S}^{1}$ in which vehicle configurations are in collision with pedestrians (yellow stars in Figure 2). This manifold is indeed computed from geometric relations between arc segments, so for every segment of the vehicle shape, we compute the intersection between the arc segment defined by $A R M_{v}$ and the approximation of $C O_{A R M}^{b}$ using (9). If such intersection point exists and belongs to both arc segments, it is considered as a candidate collision point. The point with the minimum distance (arc-length to reach that point from the origin) is the most critical one and, thus, the one to be considered. Such distance is computed as:

$$
d_{C O}(t)=\min _{i \in \mathcal{C}(t)} R_{v}(t) \delta_{i}(t)
$$

being $\mathcal{C}(t)$ the subset of candidate collision points and $\delta_{i}(t)$ their arc angle.

Due to vehicle dynamics, considering $C O_{A R M, v}$ to take decisions is in general not enough. In this sense, there exists an unsafe manifold $C U_{A R M}$, which defines the region for inevitably collisions due to vehicle dynamics [33] (stripped black region in Figure 2). Indeed, $C O_{A R M} \subset C U_{A R M}$, which contains additional vehicle configurations that are unsafe, because of the distance needed to stop the vehicle in case of emergency braking. Computing $C U_{A R M}$ for a general obstacle avoidance problem is complex as it requires to compute four additional boundary points for every boundary point of $C O_{A R M}$ [33]. Fortunately, in ADAS applications, only $C U_{A R M, v}^{b}=C U_{A R M}^{b} \cap A R M_{v}$ is of interest (red star in Figure 2), being $C U_{A R M}^{b}$ the boundary of $C U_{A R M}$. As can be seen in Figure 3, the distance of $C U_{A R M, v}^{b}$ can be computed by subtracting to $d_{C O}(t)$ the minimum distance to stop $d_{\min }(t)$ :

$$
d_{C U}(t)=d_{C O}(t)-d_{\min }(t)
$$

where $d_{\min }(t)$ will be detailed thereinafter.

Table I shows the algorithm performance to compute the $C O_{A R M}$ using our approach (arc-based) and the approach proposed in [35] (segment-based). If we assume that there are 3 shape sides (left, front, right), the number of points to compute the $C O_{A R M}$ is $N=3 \cdot 2^{n}+1$, being $n$ the number of times we split each vehicle shape side in a half. In our approach, since three points per side are considered, there is a fixed computational time with less significant error (case $n=1$ ). To obtain the accuracy achieved in our approach, the method proposed in [35] takes roughly twice to compute with $n=3^{2}$. Processing times are based on Matlab implementations using the same computer as in Section II-A.

Table I. $C O_{A R M}$ COMPUTATION: SEGMENT-BASED VS. ARC-BASED

\begin{tabular}{|l|l|c|c|c|c|c|}
\hline & $\mathbf{n}$ & $\mathbf{0}$ & $\mathbf{1}$ & $\mathbf{2}$ & $\mathbf{3}$ & $\mathbf{4}$ \\
\hline \multirow{2}{*}{$\begin{array}{l}\text { segment- } \\
\text { based }\end{array}$} & $\mathbf{t}_{\text {proc }}[\mathrm{ms}]$ & 0.348 & 0.371 & 0.516 & 0.725 & 1.157 \\
\cline { 2 - 7 } arc- & $\mathbf{e}_{C O}[\mathrm{~mm}]$ & 51.74 & 22.31 & 8.03 & 0.93 & 0.4 \\
\hline based & $\mathbf{t}_{\text {proc }}[\mathrm{ms}]$ & - & 0.313 & - & - & - \\
\cline { 2 - 7 } & $\mathbf{e}_{C O}[\mathrm{~mm}]$ & - & 1.62 & - & - & - \\
\hline
\end{tabular}

\section{Risk Evaluation}

In order to assist the speed control of the vehicles we propose the following collision risk factor to be evaluated:

$c(t)=\operatorname{sat}_{[0,1]}\left(\frac{d_{\max }(t)-d_{C O}(t)}{d_{\max }(t)-d_{\min }(t)}\right)=\operatorname{sat}_{[0,1]}\left(\frac{\Delta d(t)-d_{C U}(t)}{\Delta d(t)}\right)$

being $d_{\min }(t)$ and $d_{\max }(t)$ distance thresholds that define a spatial window $\Delta d(t)$ where the system is active:

$$
\Delta d(t)=d_{\max }(t)-d_{\min }(t)
$$

This design parameter represents the anticipation distance at which the vehicle should reduce its speed before reaching the "inevitable collision" distance $d_{\min }(t)$. Therefore, high values of $\Delta d(t)$ imply conservative solutions with anticipated warning, so the ADAS system becomes active sooner. On the contrary, low $\Delta d(t)$ values mean more aggressive solutions with sharper transition from warning to braking. As $\Delta d(t)$ is a design parameter, one can typically set it to a constant value or define an expression dependent on the vehicle velocity.

Figure 3 shows the definition of variables related to the computation of the risk factor for a frontal collision. The same concepts apply to any arbitrary $A R M$, where distances are indeed arc-lengths. A graphical example of Equation (12) is depicted in Figure 3, showing the range where the system is active: 1) if $c(t)=0$ there is no risk of collision; 2) if $c(t) \in] 0,1[$ the system is not in collision, but there is a potential risk and thus warning must be activated; 3$)$ if $c(t)=1$ there is a maximum risk of collision and therefore emergency braking must be applied to stop the vehicle.

On the one hand, our ADAS system generates a warning signal $w(t)$ based on the risk factor $c(t)$, conditioned to pressing the throttle pedal ${ }^{3}$ :

$$
w(t)= \begin{cases}c(t) & \text { if } u_{a}(t)>0 \text { or } v(t)>0 \\ 0 & \text { otherwise }\end{cases}
$$

On the other hand, if $c(t)=1$ an imminent collision is detected and then an emergency braking must be applied. In

\footnotetext{
${ }^{2}$ Error distance $e_{C O}$ is computed as the difference between $d_{C O}$ and the exact model of $C O_{A R M}$ when $n \rightarrow \infty$.

${ }^{3}$ When the driver stops pressing the accelerator pedal, we assume that he/she is aware of the danger and hence the warning signal is deactivated.
} 
such cases, an emergency signal $e(t)$ is enabled in order to stop the vehicle, although conditioned to its speed. In fact, if the velocity is higher than a given threshold $v_{e, \max }=30 \mathrm{~km} / \mathrm{h}$, the emergency braking is not activated, although the warning signal still works ${ }^{4}$ :

$$
e(t)= \begin{cases}1 & \text { if } c(t)=1 \text { and } 0<v(t)<v_{e, \max } \\ 0 & \text { otherwise }\end{cases}
$$

The minimum stop distance $d_{\text {stop }}(t)$ is dynamically computed to guarantee that the vehicle can stop by applying the maximum deceleration $a_{b, \max }(t)$ :

$$
d_{\text {stop }}(t)=0.5 \cdot v(t)^{2} / a_{b, \max }(t)
$$

where $a_{b, \max }(t)$ is obtained from (4) for $u_{b}(t)=1$.

In order to introduce an additional safety factor, we define:

$$
d_{\text {min }}(t)=d_{\text {safety }}+d_{\text {stop }}(t)
$$

where $d_{\text {safety }}$ is a safety distance where to theoretically stop the vehicle with respect to an object.

\section{Haptic Feedback Device}

Figure 4 shows a prototype of the haptic throttle used in the experimentation, which is based on a real bus pedal (MCS 962000 series). The system incorporates a motorized four-bar mechanism for blocking and ejecting the pedal by a lever when signals $w(t)$ or $e(t)$ are activated.

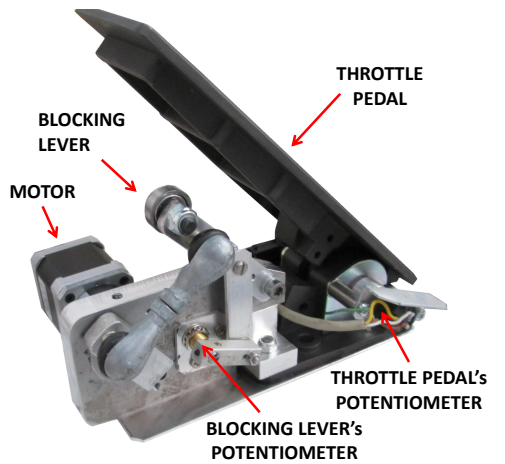

Figure 4. Haptic throttle with blocking lever based on a real bus pedal

The haptic throttle pedal provides position feedback rather than force feedback. The design of the safety system causes a kickback feeling in the driver when the warning signal is enabled, which works as a warning, not as a guidance aid. The lever is calibrated so that its angular position is linearly dependent on the warning signal $w(t)$ and the risk factor $c(t)$. Although the haptic feedback in the throttle is continuous from non-blocking position to full blocking, in order to avoid a noisy behaviour in the lever positioning, we have introduced hysteresis discretizing the range into 11 values, from $0 \%$ to $100 \%$ of blocking. In the non-blocking position the driver can freely push the pedal to the maximum position, while in the blocking position the driver must apply a force greater than $58 \mathrm{~N}$ on the throttle pedal to move the mechanism ${ }^{5}$.

\footnotetext{
${ }^{4}$ The system is designed like this in order to be consistent with current legislation for brake assist systems: Regulation UN/ECE No 13-H.

${ }^{5}$ This information has been obtained experimentally in a throttle pedal with length $0.2 \mathrm{~m}$, but it is a design parameter and might vary depending on the mechanical solution adopted.
}

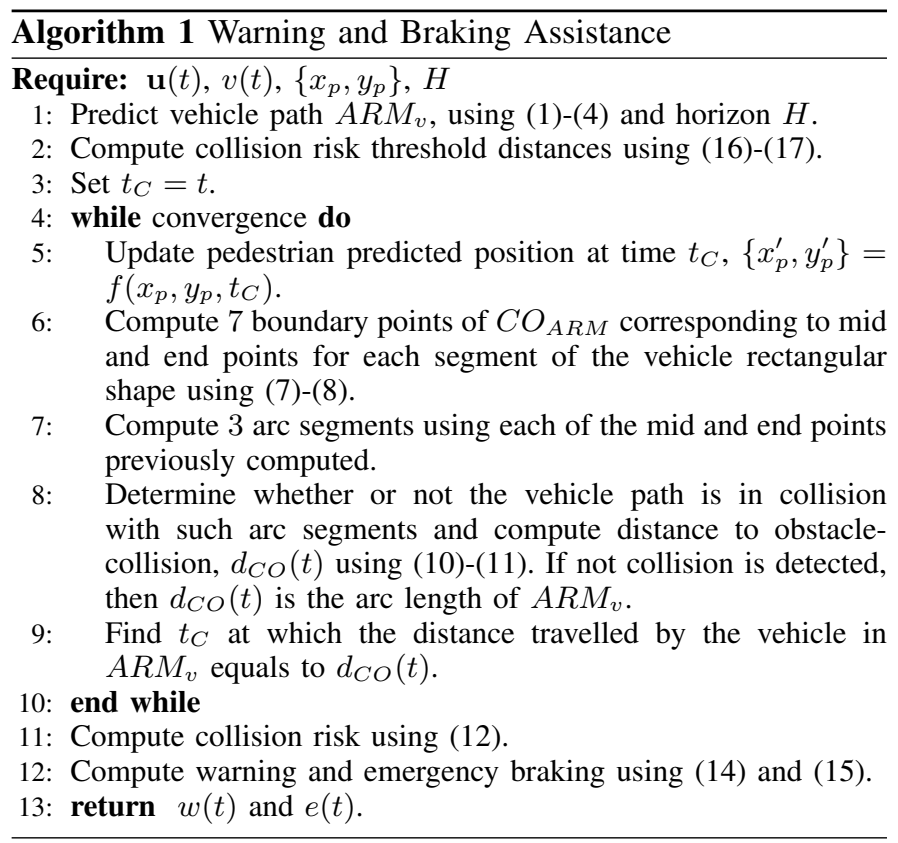

It is worth mentioning that the haptic throttle pedal has not been designed to ensure the absence of collisions, but as a warning system. When the lever tries to expel driver's foot, he/she feels a force which is reasonably enough to warn, but at the same time, one can always push it back on purpose easily (if needed). Thus, the aim is to warn the driver to improve its reaction time for braking, while making the driver feel in control of the vehicle in collision-free situations. However, the haptic pedal is not enough to avoid a collision when $e(t)=1$, so a bus specific signal is activated, which causes a maximum deceleration by applying full braking force to the wheels.

\section{E. Complete Active Safety System}

As stated in Section I, the complete ADAS proposed in this paper consists of a pedestrian detection module together with a braking assistance system with haptic feedback. The aim of this active safety system is to warn the driver and, if necessary, to stop the vehicle in dangerous manoeuvres at low speed.

The pedestrian detection system uses a set of smart cameras to detect people wandering in the vehicle surroundings. Cameras are placed in front upper part of the bus (left, center and right) pointing downwards, covering the periphery of the bus from an overhead view, and an additional camera pointing to the front, able to detect pedestrians from a greater distance. To know driver's intention the ADAS requires additional sensing such as a potentiometer in the steering column and potentiometers on throttle and brake pedals. Further details on implemented pedestrian detection system can be found in [36].

In this contribution, let's assume that motions $\left\{x_{p}^{\prime}, y_{p}^{\prime}\right\}=$ $f\left(x_{p}, y_{p}, t\right)$ can be predicted for every pedestrian from a smart camera. Algorithm 1 shows an iterative procedure to determine collision risk factor $c(t)$, Equation (12), for a given prediction horizon $H$ and for each moving pedestrian. The processing time of a $\mathrm{C}++$ implementation of the proposed algorithm is $t_{\text {proc }}=0.15 \pm 0.05 \mathrm{~ms}$, using the same PC as in Section II-A. 


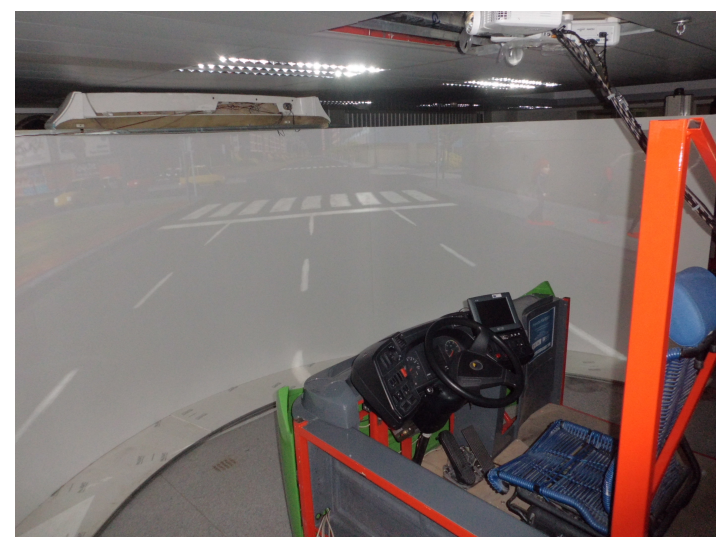

Figure 5. Driving simulation cabin with projection system composed by 3 projectors and a semicircular screen.

\section{EXPERIMENTAL SETUP}

A driving simulation cabin was used as a tool to evaluate the performance of the proposed ADAS. Several experiments have been carried out in order to validate the behaviour of the braking assistance system with haptic feedback on a simulated environment. As shown in Figure 5, the simulation platform includes the following devices: a driving cabin with a seat, a steering wheel, pedals and a gearshift; and a projection system composed by three synchronised video projectors for environmental simulation onto a semicircular screen. Regarding the simulation engine, it is based on PhysX SDK (version 3.3.1) for computation of rigid body dynamics and collisions, while OGRE (version 1.8.1) is used as graphics engine for rendering scenarios and visualization.

The vehicle type used for experimentation is a bus with the following parameters: mass $m=10630 \mathrm{~kg}$ (tare or unladen weight), length $l=7 \mathrm{~m}$, width $w=2.6 \mathrm{~m}$, axles distance $L=6 \mathrm{~m}$ and maximum turning angle $\alpha_{\max }=\pi / 4 \mathrm{rad}$. After identification using the step response method, we obtained a first-order model as in (2), with $K=16.81, \tau=12.47 \mathrm{~s}$ and $\tau_{d}=0.1 \mathrm{~s}$. On the other hand, the braking model in (4) was approximated using the following values: $k_{0}=0, k_{1}=-0.03$, $k_{2}=-5.97, k_{3}=4.41 \cdot 10^{-4}, k_{4}=1.79$ and $k_{5}=0$, with a maximum deceleration of $a_{b, \max } \approx-4.5 \mathrm{~m} / \mathrm{s}^{2}$.

Unless specified otherwise, in all tests the parameters for the ADAS were set to the following values: safety distance $d_{\text {safety }}=1 \mathrm{~m}$ and $\Delta d=10 \mathrm{~m}$, with $R_{p}=0.3 \mathrm{~m}$ as a constant radius to define pedestrians shape.

\section{A. Emergency braking: mass influence}

The aim of this experiment is to evaluate the robustness of the emergency braking system against modelling errors, for different bus loads (assuming a capacity of 58 passengers and approximately $70 \mathrm{~kg}$ per passenger): $m=11000 \mathrm{~kg}$ (empty, with kerb weight), $m=13000 \mathrm{~kg}$ (half loaded) and $m=15000$ $\mathrm{kg}$ (full loaded). In this test, the pedestrian is placed initially at a distance of $d_{C O}=40 \mathrm{~m}$ far from the bus. Then the driver starts pressing the throttle and the vehicle speeds up and moves straight forward against the obstacle. The haptic warning is disabled to evaluate only the emergency braking effect. Indirectly, we also want to show how $d_{\text {safety }}$ can be obtained to guarantee the absence of collision even when the vehicle model has uncertainties.

\section{B. Haptic warning: distance range influence}

In order to evaluate the influence of the distance range $\Delta d$ in which the warning is active, we performed a series of 7 tests varying from $\Delta d=0 \mathrm{~m}$ to $\Delta d=30 \mathrm{~m}$, with increments of $5 \mathrm{~m}$. Initially, the pedestrian was placed at $d_{C O}=40 \mathrm{~m}$, the bus was half loaded $m=13000 \mathrm{~kg}$ and the safety distance was set to $d_{\text {safety }}=1 \mathrm{~m}$. The driver had to proceed as in the previous experiment, but in this case reacting as soon as he felt the haptic feedback in the throttle. This experiment also shows how to get an appropriate value of $\Delta d$, justifying the value used in the experimentation carried out in Section IV-D.

\section{Haptic warning: driving behaviour influence}

Through this experimentation, we evaluate the influence of the haptic feedback under different driving behaviours or styles, from expected to aggressive. As in the previous test, the vehicle moves forward against a static pedestrian placed at $d_{C O}=14 \mathrm{~m}$ far from the front of the bus. The driver was addressed to brake the vehicle when considered necessary. In the expected behaviour the driver reacted as soon as he felt the haptic feedback. In the second attempt, the subject was addressed to drive in a more aggressive way, without taking the haptic warning into account. In this experiment the bus was also half loaded $m=13000 \mathrm{~kg}$.

\section{Benchmarking evaluation}

The last set of experiments involved 20 different drivers (19 males and 1 female with ages between 18 and 55, mean age $30 \pm 5.6$ ), each one driving for about half an hour around an urban scenario. A specific area of the city of Valencia (Spain) has been modelled, reproducing very realistic driving conditions including traffic and pedestrian logic. Even though users were not expert bus drivers, all of them had driving licence (experience of $8 \pm 3.4$ years in average) and used to drive their car frequently. Before starting, the subjects experienced the driving simulator for as long as they needed, in order to get used to it and to the urban circuit. The experimentation consisted of taking 4 loops to a circuit composed by 6 bus stops. The bus route was more than $2.2 \mathrm{~km}$ long, so each subject drove for about $9 \mathrm{~km}$ and did 24 bus stops.

During the test, drivers were addressed to drive normally around the city, following a given path and stopping at all bus stops. When the vehicle was approaching or leaving a bus stop, randomly a pedestrian showed up walking in front of the bus. Drivers did not know when or where pedestrians showed up. In order to counterbalance the cases with/without the safety system active, among all risky situations, half the times the haptic feedback and the emergency braking were disabled, always randomly. Furthermore, in order to avoid a "learning effect" produced by the drivers getting used to the experiment, pedestrians appeared in a random pattern before or after a bus stop, in both cases or in none of them. Besides, risky situations were designed so that it was possible to stop the vehicle in time. For instance, pedestrians did not show up if the vehicle was moving too fast in an approach manoeuvre 


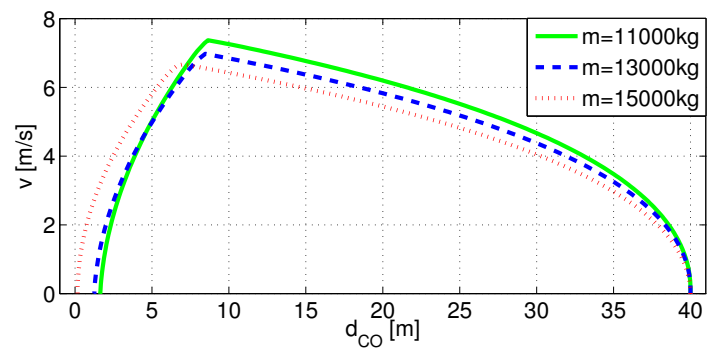

Figure 6. Phase diagram of distance $d_{C O}(t)$ versus velocity $v(t)$ for different values of vehicle mass $m$.

because the accident would be unavoidable. On the other hand, to avoid that participants were able to predict a pedestrian walking in front of the bus, those pedestrians used specifically in risky situations were transparent and became visible when the incident was produced. Hence, situations in which the driver is distracted or his view is obstructed were reproduced.

The aim of such experiment was to compare the performance when the safety system was enabled against cases in which it was disabled. Several aspects will be analysed in Section IV-D, such as percentages of low, medium, high risk situations and collisions, as well as driver's reaction time in warning situations and time to collision. In the experimentation, we consider the following risk conditions:

- Low: when $d_{\min }<d_{C O} \leq d_{\max }$.

- Medium: when $d_{\text {safety }}<d_{C O} \leq d_{\text {min }}$.

- High: when $0.1 \mathrm{~m}<d_{C O} \leq d_{\text {safety }}$ and $v>1.5 \mathrm{~m} / \mathrm{s}$.

- Collision: when $d_{C O} \leq 0.1 \mathrm{~m}$ and $v>0.6 \mathrm{~m} / \mathrm{s}$.

On the other hand, in order to measure driver's time of reaction $t_{R}$, we compute the delay between the instant when the haptic feedback starts warning through the blocking lever and the instant when the driver presses the brake at least a $10 \%$ of its range. Time to collision $t_{C}$ is computed recursively, as explained in Algorithm 1. For this analysis, we distinguish between situations where the bus slows down to stop (approach manoeuvres), from those in which the bus starts its movement (departure manoeuvres). It must be remarked that the collision risk evaluation system is computed in any case, with the ADAS enabled or disabled, in order to compare the performance.

\section{RESULTS}

\section{A. Emergency braking: mass influence}

This set of tests shows the influence of bus mass in an emergency braking and how $d_{\text {safety }}$ can be set to guarantee no collision even with modelling errors. In this experiment we used a model of the bus half loaded, considering a mass of $m=13000 \mathrm{~kg}$. As the safety distance was set to $d_{\text {safety }}=1 \mathrm{~m}$, the vehicle should stop at such distance far from the obstacle (dashed blue line in Figure 6). However, if the model is not accurate enough, the vehicle does not behave as expected, braking before or after it should (green and dotted red lines in Figure 6, respectively). In the worst case scenario, we would be using a model of the bus completely empty for the collision detection, when in fact it would be full. This would imply braking too late in some situations and therefore adding an extra safety distance is necessary to avoid crashes. Such distance $d_{\text {safety }}$ should be at least the difference between the
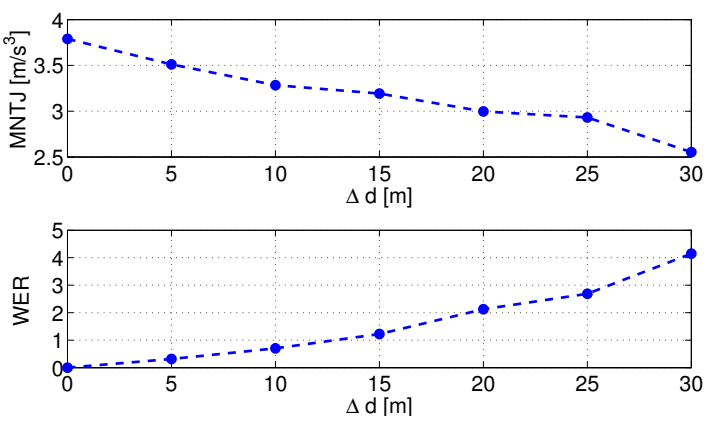

Figure 7. Mean Normalized Total Jerk $M N T J$ and Warning-Emergency Ratio $W E R$ for different values of $\Delta d$.

distance travelled by the vehicle (moving approximately at the maximum speed at which the emergency braking works) when it is full loaded and when it is with the same load as the model used in the collision detection.

\section{B. Haptic warning: distance influence}

In this experimentation, the influence of the variation in distance $\Delta d$ is evaluated. To do so, we have used the following longitudinal metrics: Mean Normalized Total Jerk (MNTJ) and Warning-Emergency Ratio (WER).

On the one hand, MNTJ takes into account changes in acceleration or deceleration and is defined as:

$$
M N T J=\frac{1}{N} \sum_{i=0}^{N}\left|\frac{d a_{x, i}}{d t}\right|
$$

being $a_{x, i}$ the longitudinal acceleration.

On the other hand, $W E R$ considers the ratio between the distance travelled in a warning situation $d_{\text {warning }}$ and the distance travelled when an emergency braking is produced $d_{\text {emergency }}$.

$$
W E R=\frac{d_{\text {warning }}}{d_{\text {emergency }}}
$$

Figure 7 shows the results obtained for different values of $\Delta d$. It can be observed that the shorter $\Delta d$ is the higher the $M N T J$ and the sharper the emergency braking. In the limit of $\Delta d=0 \mathrm{~m}$, there is no warning at all as $W E R=0$ and therefore there is no transition between a non-collision situation and an emergency situation. We consider that a $W E R \approx 1$ is good, because lower values imply more aggressive safety actions without warning, while values too big are annoying to the driver as the system starts working when the pedestrian is still too far away. In this sense, we chose $\Delta d=10 \mathrm{~m}$ for the experimentation carried out in Section IV-D.

\section{Haptic warning: driving behaviour influence}

Figure 8 shows two cases representing different driver's behaviour: expected and aggressive. In Figure 8(a), in the first case (red line) the driver intentionally does not react when the blocking lever tries to eject the throttle so he/she keeps pressing it, let's define that as aggressive driving. The second situation (dashed blue line) is an expected behaviour that happens when the driver reacts immediately releasing completely the throttle after feeling some force in the pedal. Obviously, between this 


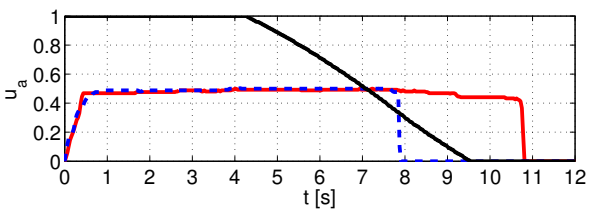

(a) Throttle position vs. Inverted warning signal

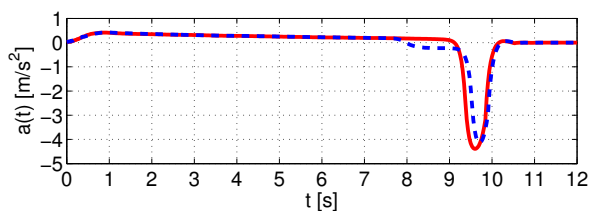

(b) Acceleration

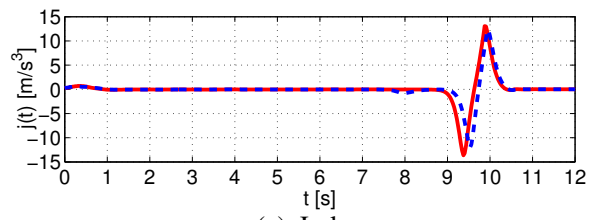

(c) Jerk

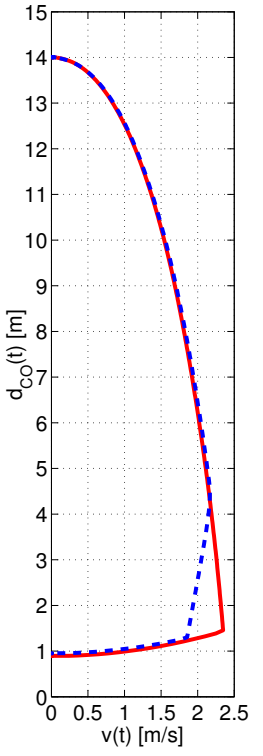

(d) Phase diagram

Figure 8. Experiments with two opposite driver's reaction to the haptic feedback device: aggressive (red) and expected (dashed blue). Black line represents the allowed range to press the gas pedal, computed as $b(t)=1-w(t)$. In both cases the vehicle moves forward against an obstacle placed at $d_{C O}=14 \mathrm{~m}$.

two extreme situations there is a range of behaviours depending on driver's time of reaction after feeling the haptic feedback.

On the other hand, in Figures 8(b) and 8(c) longitudinal acceleration and jerk are depicted, respectively. In the instant $t \approx 8 \mathrm{~s}$ is when the driver stops pressing the throttle in the expected behaviour (dashed blue line), which implies a slight negative deceleration before the emergency braking is produced at $t \approx 9 \mathrm{~s}$. This behaviour implies that the maximum jerk suffered by the vehicle is lower and therefore more comfortable than the one produced by a more aggressive driving (red line). It is interesting to remark the equivalence between aggressive driving and a more conservative driving without haptic warning, i.e. with $\Delta d=0 \mathrm{~m}$. In fact, in both situations the emergency braking is applied directly without the driver reacting previously.

Finally, Figure 8(d) depicts the phase diagram of the proposed deceleration control law in order to analyse the performance of the safety system under each driver behaviour. The abscissa axis represents vehicle velocity, while the ordinate axis represents the distance of the vehicle to collide (without taking into account the length of the bus, since distances are measured from its front part). Initially, the pedestrian is placed at $d_{C O}=14 \mathrm{~m}$ far from the vehicle. Then the driver starts pressing the throttle and the vehicle speeds up. When the obstacle is at $d_{C O}<d_{\max }$ the blocking system becomes active and ejects the throttle pedal. Finally, as soon as $d_{C O}<d_{\min }$ an emergency braking is applied guaranteeing that when the bus is fully stopped the pedestrian is at a distance around $d_{\text {safety }}$ far from the front part of the vehicle. It can be seen that the proposed control law cancels out driver actions and safely stops the vehicle before crashing in all considered situations. Indeed the safety system is effective even in cases of aggressive driving or with late reactions.

\section{Benchmarking evaluation}

The experimentation carried out produced an amount of 606 risky situations, half of the incidents with the system disabled and the other half with it enabled. When the system was disabled there was a $59.08 \%$ of low risk cases, while the remaining $40.92 \%$ of incidents had medium risk $(27.06 \%)$, high risk $(13.2 \%)$, or even collisions $(0.66 \%)$. On the contrary, when the system was enabled, even though the proportions were similar in terms of low risk cases with a $60.73 \%$, in the remainder there were no collisions and the high risk situations were reduced to a mere $3.63 \%$. This phenomena can be attributed to the emergency braking, which is able to stop autonomously the vehicle in time in situations in which the driver would not be fast enough.

In order to highlight the benefits provided by the warning system, we will evaluate the driver's reaction time $t_{R}$ in low risk cases. In our experimentation, the time of reaction was measured as the delay between the instant in which a pedestrian appears and the time instant when the driver presses the brake pedal. Empirically we obtained that $t_{R}$ varies from $0.3 \mathrm{~s}$ to $1.2 \mathrm{~s}$. We discarded values of $t_{R}<0.3 \mathrm{~s}$ because either the driver was already pressing the brake pedal or we consider that driver's intention was to brake before even seeing any potential collision.

In Table II we analyse the incidents when the vehicle is approaching the bus stop (STOP) and when it is departing (START). In both cases we compare the results when the safety haptic system is disabled (OFF) and when it is enabled (ON). It can be seen that the haptic feedback does not affect much the results in approach manoeuvres, as both populations are not very different, although the time of reaction is slightly faster when the system is enabled (ON). Indeed, when the vehicle is approaching to a bus stop and an emergency situation occurs, the average reaction time is $t_{R} \approx 0.59 \mathrm{~s}$ if the haptic feedback is OFF and $t_{R} \approx 0.54 \mathrm{~s}$ when it is ON. In both cases the standard deviation is very similar $\sigma \approx 0.25 \mathrm{~s}$.

Table II. REACTION TIME $t_{R}$ IN APPROACH (STOP) AND DEPARTURE

(START) MANOEUVRES, WITH HAPTIC THROTTLE AND EMERGENCY BRAKING DISABLED (OFF) AND ENABLED (ON).

\begin{tabular}{|l|c|c|}
\hline & STOP & START \\
\hline OFF & $0.5927 \pm 0.2667 \mathrm{~s}$ & $0.7026 \pm 0.1875 \mathrm{~s}$ \\
\hline ON & $0.5423 \pm 0.2434 \mathrm{~s}$ & $0.5887 \pm 0.1805 \mathrm{~s}$ \\
\hline
\end{tabular}

On the other hand, when the vehicle departs from a bus stop and a potential collision is detected the average reaction time is $t_{R} \approx 0.7 \mathrm{~s}$ when the system is disabled (OFF), while it is $t_{R} \approx 0.59 \mathrm{~s}$ when it is enabled $(\mathrm{ON})$. Once more, the standard deviation is very similar in both cases $\sigma \approx 0.18 \mathrm{~s}$. So, the haptic warning helps the driver be approximately $17 \%$ faster in pressing the brake in situations with low risk of collision.

Now, in order to check the validity of results we have performed one-way analysis of variance (ANOVA) to evaluate the factor haptic feedback. The purpose is to determine whether data from the groups (levels) of the factor haptic feedback, which are $\mathrm{OFF}$ and $\mathrm{ON}$, have a common mean or, on the contrary are significantly different. From ANOVA tests we get that when the vehicle approaches the bus stop the $p$ value is $p=0.382$. So, we cannot reject the null hypothesis 
at the 0.05 significance level. Hence we conclude that the observed differences are not statistically significant. So the haptic feedback does not improve safety when the vehicle approaches the bus stop. On the other hand, in departure manoeuvres after ANOVA analysis we get that $p=1.227 \cdot 10^{-7}$. Therefore, we reject the null hypothesis and can conclude that both groups of data are statistically different with $5 \%$ of significance. Hence, the haptic feedback reduces driver's time of reaction $t_{R}$ in departure manoeuvres.

In addition to this, the proportion of low and medium risk incidents is higher when the safety warning is enabled. Since the haptic feedback reduces the reaction time, the expected mean velocity in these situations is also reduced. Mean velocity is measured from the instant when the risk is detected to the instant in which it disappears, because either the vehicle stops or speed is reduced so that there is no risk any more. We obtained, from these experiments, a mean velocity value of $\bar{v}=2.8 \mathrm{~m} / \mathrm{s}$ when the system is enabled compared to $\bar{v}=4.5$ $\mathrm{m} / \mathrm{s}$ when the system is disabled in those situations. Therefore, we can imply that safety is also improved as the severity in case of collision is reduced by reducing the speed of the vehicle.

Finally, we analyse the time to collision $t_{C}$ in emergency situations (corresponding to cases of medium risk, high risk or collision) in order to highlight the benefits of the autonomous braking. After analysing the results, it can be concluded that the time to collision when the safety system is enabled (ON) is bigger $\left(t_{C}=0.8648 \pm 0.4268 \mathrm{~s}\right)$ than when it is disabled (OFF) $\left(t_{C}=0.7086 \pm 0.3309 \mathrm{~s}\right)$. The standard deviation has also been reduced considerably. Furthermore, in the obtained results there are no cases with $t_{C}<0.2 \mathrm{~s}$ with the emergency braking enabled. This means that the use of the proposed system increases safety as long as the probability distribution of the time to collision has been moved away from $t_{C}=0 \mathrm{~s}$.

However, to prove the validity of the results we have performed an ANOVA test. The results imply that both populations, when the factor emergency braking takes its two possible levels $\mathrm{OFF} / \mathrm{ON}$, are statistically different with a significance of $5 \%$, as the statistic $p=0.001$. Therefore, we state that the observed improvements in the time to collision are due to the usage of the proposed active safety system.

\section{DISCUSSION}

As stated in Section I, the main contribution of this paper is the development and testing of a new ADAS to help bus drivers in low-speed manoeuvres in urban environments, mostly in areas of passenger loading and unloading. The novelty lies in the fact that, after doing our own research and among all the papers cited in the extensive survey carried out by Petermeijer et.al. in [30], we did not find any approach using a haptic throttle pedal in order to avoid crashes with pedestrians.

Following the classification developed in [30], we consider that the proposed safety system for bus braking assistance working at low speed to avoid collisions with pedestrians could be described as a warning system with automatic emergency braking (function level), intended for collision avoidance and speed adaptation at a manoeuvring level (task level), that uses a haptic throttle (channel level) applying a counterforce to communicate with the driver (signal level).
In [25]-[28], authors use active gas pedals applying a counterforce when the speed limit is exceeded. This is very similar to what is done in the present paper, although applied to "safe speed and safe distance" (SASPENCE). In this sense, our findings are consistent and also show a statistically significant reduction in driver's reaction time when the haptic warning is enabled. Furthermore, it has been also shown that a haptic throttle pedal can help to reduce the number of high risk incidents and collisions.

In other works, haptic warnings are used to avoid rearend collisions with vehicles using vibro-tactile and/or audiovisual feedback [17]-[19]. Similar works were carried out for collision avoidance problems using a haptic seat [13], a haptic steering wheel [14] or auditory feedback and a haptic pedal [22], [25]. However, only two studies have been found regarding collision avoidance with pedestrians. They use either auditory and tactile feedback [11] or haptic steering guidance [12]. But, none of them evaluates the effect of an active gas pedal as a warning to reduce the risk of collision with pedestrians. Therefore, our approach is a new contribution in the field as there are no previous solutions to this problem.

As in most of the mentioned papers, our findings prove that the active throttle with haptic feedback reduces driver's reaction time, so the average speed is slowed and the time to collision increased, which indirectly decreased the number of medium and high risk situations in which an emergency braking is necessary. Moreover, we have also shown that using haptic warnings reduce the abruptness when braking and therefore injuries occurred on board are decreased. On the other hand, in medium or high risk incidents the emergency braking is an extra safety device able to reduce the speed automatically when the driver does not react in time, which helps to reduce the number of crashes. Therefore, the improvement in safety is due to the combination of both parts, although it can be concluded that the automatic emergency braking is the ultimate responsible for reducing the number of accidents.

Among the limitations of the proposed method, we think that the simplified model of the vehicle (acceleration and braking) is very useful to check for collisions very quickly. However, as it is only valid for a vehicle and a kind of surface, the system could be improved by identifying models for different masses (loads), contact surfaces, among other variants.

The implementation of the proposed active safety system into city buses is not straightforward, requiring compliance with current legislation and relevant approvals. Regulations UN/ECE 13 and UN/ECE 131 affect vehicle's emergency braking, which might imply its integration within the Electronic Braking System (EBS). Regarding the haptic throttle pedal, the system does not affect the original vehicle configuration. Besides, as far as we know there is no-regulation regarding the maximum force that can be applied to the gas pedal, it only depends on the bus manufacturer. All these regulation aspects have been taken into account during the development of our preliminary pedestrian detection and emergency brake system in the context of the SAFEBUS project, see [36] for further details. As a result of the project we were able to perform some preliminary tests using a real bus, which constituted a proof of concept for the proposed systems in this paper. 


\section{CONCLUSIONS}

A new advanced outdoor safety system for buses in urban environments at low speeds has been introduced and tested. The proposed approach is based on a computationally efficient algorithm that uses a simplified model of vehicle dynamics. This model has been proven to be applicable to collision detection with similar performance to more complex models, but with less processing load. The algorithm considers driver's intention, vehicle shape and dynamics in order to evaluate the risk of collision with pedestrians moving around the vehicle. Moreover, a haptic feedback device has been developed to warn the driver in case of risk of collision, which consists of an active throttle pedal with a blocking mechanism. Under imminent collision conditions, the safety system sends an electric signal to enable the emergency braking system.

In the article, we have analysed the influence of the main parameters affecting the proposed method. Besides, the effectiveness of the developed safety system has been tested through some experiments carried out using a real prototype of the haptic throttle in a driving simulator. These experiments demonstrate that the haptic feedback in the throttle pedal is able to warn and interact with the driver when a dangerous manoeuvre happens, which reduces the reaction time and the number of situations in which the emergency braking system gets active. If a dangerous manoeuvre persists, the emergency braking guarantees collision-free situations, which is an improvement in safety.

As further work, we aim to validate the complete safety system in a real scenario, using an urban transport bus. Thus, the effectiveness of the haptic feedback could be analysed in depth with professional bus drivers. Moreover, we also want to enhance the haptic warning using audio-visual feedback devices, analysing their effects individually and together.

\section{ACKNOWLEDGEMENTS}

This paper has been funded by Ministerio de Ciencia e Innovación (Spain) through the projects "Sistemas Avanzados de Seguridad Integral en Autobuses (SAFEBUS)" (IPT-2011-1165-370000) and "Sistemas de Conducción Segura de Vehículos de Transporte de Pasajeros y Materiales con Asistencia Háptica/Audiovisual e Interfaces Biomédicas (SAFETRANS)" (DPI2013-42302-R). This work was also supported by Programa VALi+d (Generalitat Valenciana). The authors wish to thank José Luís Sánchez Carrascosa for his commitment to the project. Finally, thanks to Ana Isabel Sánchez Galdón for her valuable help regarding ANOVA analysis.

\section{REFERENCES}

[1] WHO, "Global status report on road safety 2015," World Health Organization, Tech. Rep., 2015.

[2] ASCABUS, "El sector carrocero de autobuses y autocares y el transporte de viajeros en cifras," Asociación Española de Carroceros de Autobuses y Autocares, Tech. Rep., 2013.

[3] CARE Database, "Traffic safety basic facts: Heavy goods vehicles and buses," European Road Safety Observatory, Tech. Rep., 2015.

[4] M. Seguí, "Main figures on road safety data: Spain 2013," Dirección General de Tráfico (DGT), 2014.

[5] M. Berntman, A. Wretstrand, and B. Holmberg, "Bus travel safety - a travel chain perspective," in International conference on Mobility and Transport for Elderly and disabled Persons, 2010, p. 10.
[6] NHTSA's National Center for Statistics and Analysis, "Schooltransportation-related crashes," National Highway Traffic Safety Administration (USA), Tech. Rep., 2014.

[7] D. Racine, N. Cramer, and M. Zadeh, "Active blind spot crash avoidance system: A haptic solution to blind spot collisions," in Haptic AudioVisual Environments and Games (HAVE), 2010 IEEE International Symposium on, Oct 2010, pp. 1-5.

[8] J. Morrell and K. Wasilewski, "Design and evaluation of a vibrotactile seat to improve spatial awareness while driving," in Haptics Symposium, 2010 IEEE, March 2010, pp. 281-288.

[9] J. Chun, I. Lee, G. Park, J. Seo, S. Choi, and S. H. Han, "Efficacy of haptic blind spot warnings applied through a steering wheel or a seatbelt," Transportation Research Part F: Traffic Psychology and Behaviour, vol. 21, pp. $231-241,2013$.

[10] J. J. Scott and R. Gray, "A comparison of tactile, visual, and auditory warnings for rear-end collision prevention in simulated driving," Human Factors, vol. 50, no. 2, pp. 264-275, May 2008.

[11] S. M. Straughn, R. Gray, and H. Z. Tan, "To go or not to go: Stimulusresponse compatibility for tactile and auditory pedestrian collision warnings," IEEE Transactions on Haptics, vol. 2, no. 2, pp. 111-117, Apr. 2009.

[12] M. Itoh, T. Inagaki, and H. Tanaka, "Haptic steering direction guidance for pedestrian-vehicle collision avoidance," in Systems, Man, and Cybernetics (SMC), 2012 IEEE International Conference on, Oct 2012, pp. $3327-3332$.

[13] G. M. Fitch, J. M. H. amd Brian M. Kleiner, and T. A. Dingus, "Driver comprehension of multiple haptic seat alerts intended for use in an integrated collision avoidance system," Transportation Research Part F: Traffic Psychology and Behaviour, vol. 14, no. 4, p. 278-290, July 2011.

[14] M. J. Jensen, A. M. Tolbert, J. R. Wagner, F. S. Switzer, and J. W. Finn, "A customizable automotive steering system with a haptic feedback control strategy for obstacle avoidance notification," IEEE Transactions on Vehicular Technology, vol. 60, no. 9, p. 4208-4216, 2011.

[15] C. Wu, L. Peng, Z. Huang, M. Zhong, and D. Chu, "A method of vehicle motion prediction and collision risk assessment with a simulated vehicular cyber physical system," Transportation Research Part C, vol. 47, pp. 179-181, 2014.

[16] T. Dunn, R. Laver, D. Skorupski, and D. Zyrowski, "Assessing the business case for integrated collision avoidance systems on transit buses. fta-omb no. 0704-0188," US Department of Transportation, Tech. Rep., 2007.

[17] C.-Y. Chang and Y.-R. Chou, "Development of fuzzy-based bus rearend collision warning thresholds using a driving simulator," Intelligent Transportation Systems, IEEE Transactions on, vol. 10, no. 2, pp. 360365, June 2009.

[18] T. Kim and H.-Y. Jeong, "Crash probability and error rates for headon collisions based on stochastic analyses," Intelligent Transportation Systems, IEEE Transactions on, vol. 11, no. 4, pp. 896-904, Dec 2010.

[19] R. Mohebbi, R. Gray, and H. Z. Tan, "Driver reaction time to tactile and auditory rear-end collision warnings while talking on a cell phone," Human Factors, vol. 51, no. 1, pp. 102-110, February 2009.

[20] Z. Zheng, S. Ahn, D. Chen, and J. Laval, "The effects of lane-changing on the immediate follower: Anticipation, relaxation, and change in driver characteristics," Transportation Research Part C, vol. 26, pp. 367-379, 2013.

[21] S. de Nijs, M. Mulder, and D. Abbink, "The value of haptic feedback in lane keeping," in Systems, Man and Cybernetics (SMC), 2014 IEEE International Conference on, Oct 2014, pp. 3599-3604.

[22] E. Adell, A. Várhelyi, M. Alonso, and J. Plaza, "Developing humanmachine interaction components for a driver assistance system for safe speed and safe distance," IET Intelligent Transport Systems, vol. 2, no. 1 , pp. 1-14, 2008

[23] M. Mulder, J. Pauwelussen, M. van Paassen, M. Mulder, and D. Abbink, "Active deceleration support in car following," Systems, Man and 
Cybernetics, Part A: Systems and Humans, IEEE Transactions on, vol. 40, no. 6, pp. 1271-1284, Nov 2010.

[24] M. Mulder, D. Abbink, M. van Paassen, and M. Mulder, "Design of a haptic gas pedal for active car-following support," Intelligent Transportation Systems, IEEE Transactions on, vol. 12, no. 1, pp. 268279, March 2011.

[25] E. Adell, A. Várhelyi, and M. Hjälmdahl, "Auditory and haptic systems for in-car speed management - a comparative real life study," Transportation Research Part F, vol. 11, no. 1, pp. 445 - 458, 2008.

[26] E. Adell, A. Várhelyi, and M. dalla Fontana, "The effects of a driver assistance system for safe speed and safe distance - a real-life field study," Transportation Research Part C: Emerging Technologies, vol. 19, no. 1, pp. 145 - 155, 2011.

[27] A. Várhelyi, M. Hjälmdahl, C. Hydén, and M. Draskóczy, "Effects of an active accelerator pedal on driver behaviour and traffic safety after longterm use in urban areas," Accident Analysis and Prevention, vol. 36, no. 1, pp. 729-737, 2004.

[28] M. Hjälmdahl and A. Várhelyi, "Speed regulation by in-car active accelerator pedal. effects on driver behaviour," Transportation Research Part F, vol. 7, no. 1, pp. 77-94, 2004.

[29] E. Adell and A. Várhelyi, "Driver comprehension and acceptance of the active accelerator pedal after long-term use," Transportation Research Part F, vol. 11, no. 1, pp. 37-51, 2008.

[30] S. M. Petermeijer, D. A. Abbink, M. Mulder, and J. C. F. de Winter, "The effect of haptic support systems on driver performance: A literature survey," IEEE Transactions on Haptics, 2015.

[31] T. D. Gillespie, Fundamentals of Vehicle Dynamics. Society of Automotive Engineers, 1992.

[32] H. B. Pacejka, Tire and Vehicle Dynamics, 2nd edition. SAE International, 2005.

[33] J. Minguez and L. Montano, "Extending collision avoidance methods to consider the vehicle shape, kinematics, and dynamics of a mobile robot," IEEE Transactions on Robotics, vol. 25, no. 2, pp. 367-381, 2009.

[34] G. Campion, G. Bastin, and B. Dandrea-Novel, "Structural properties and classification of kinematic and dynamic models of wheeled mobile robots," IEEE Transactions on Robotics and Automation, vol. 12, no. 1, pp. 47-62, 1996.

[35] J. Minguez, L. Montano, and J. Santos-Victor, "Abstracting vehicle shape and kinematic constraints from obstacle avoidance methods," Autonomous Robots, vol. 20, no. 1, pp. 43-59, 2006.

[36] L. Armesto, L. Arnal, J. Dols, V. Girbés, and J. C. Peris, "Safebus project: Advanced safety systems in buses," RIAI (In press), pp. 1-12, 2015.

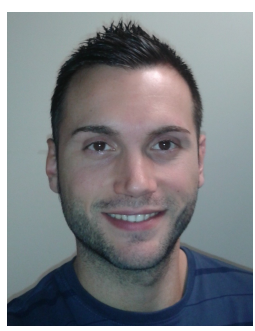

Vicent Girbés received the B.Eng. (Hons) in Electronics and Control in 2009 and the M.Sc. in Control and Computer Science in 2010, both from the Universitat Politècnica de València (UPV), Spain. He was awarded 1st Best B.Eng. National Prize from the Spanish Ministry of Education, and also with an Academic Performance Award from the Valencian Government (GVA). In 2009 Mr. Girbés joined the Robotics and Automation Research Group of the Design and Manufacturing Institute (IDF-UPV). From 2010 to 2014 he held a former researcher fellowship in the VALi+d Program (GVA). In 2013 he was visiting researcher at the Control Systems Group of the University of Manchester (UK). From 2015 he has been working on two Spanish national projects: SAFEBUS and SAFETRANS. In 2016 he obtained the Ph.D. degree for his thesis about smooth path planning and control in intelligent vehicles, applied to autonomous and manual-assisted driving. Fields such as computer vision, mobile robotics and driver assistance through haptic and audiovisual feedback are also among his interests.

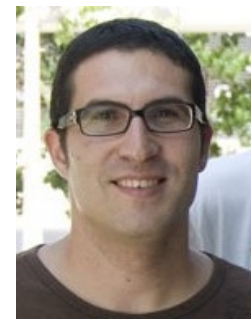

Leopoldo Armesto received the B.Sc. degree in Electronic Engineering, the M.Sc. degree in Control Systems Engineering, and the Ph.D. in Automation and Industrial Computer Science from the Universitat Politècnica de València (UPV), Spain, in 1998, 2001, and 2005, respectively. He held a Ph.D. Scholarship for three years at the Department of Systems Engineering and Control at the same University, where he is Assistant Professor since 2004. He is currently member of the Robotics and Automation Research Group of the Design and Manufacturing Institute (IDF-UPV). His current research interests are Mobile Robotics, Optimal Control, Advanced Driving Assistance Systems, 3D Printing and Reinforcement Learning. He has supervised 2 Ph.D. theses and 6 final M.Sc. Projects. He has participated in 20 Research Projects (leading 6 of them) and many other contracts with industry. He has published $8 \mathrm{JCR}$ journal papers and 60 conference papers (google h-index 10).

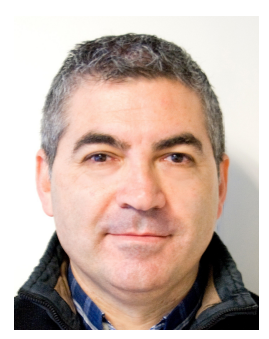

Juan Dols is Industrial Engineer (Mechanics) since 1987 and in 1996 the Ph.D. degree at Technical University of Valencia-UPV (Spain), where he is now teaching Automotive and Transportation Engineering at the Mechanical and Materials Engineering Department of UPV. Professor Dols is also Director of the UPV Automobile Laboratory (LAUPV). His research activity is based in the design and development of driving simulators, wheelchair tiedown and occupant restraint systems, active and passive safety in vehicles, accessible transportation engineering, driving aids design and development, accident reconstruction technologies and vehicle dynamics. He has conducted as main researcher and coordinator several national projects (SEMAV, SATRUS, DGT, DATOS, ASUCAR, SE2RCO) and international EU funded projects (FORCE, ADAPT, TRAINER, CONSENSUS, IDEA, ASK-IT) in the 4th, 5th and 6th framework EU research programs. He also serves in the Editorial Board of the "Securitas Vialis" Journal, and has participated as reviewer in "Assistive Technology" and "Ergonomics" journals. Professor Dols has been Chairman of working group ISO TC22/WG4 "Accessibility in Public Transport Vehicles", and member of ISO TC173/SC1/WG6 "Restraint Systems for the Transport of Handicapped People in Wheelchairs", and full member of SAE, STA, EMG professional associations.

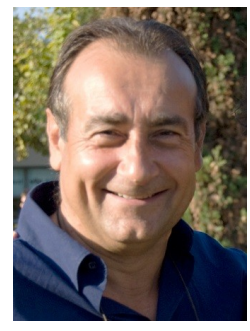

Josep Tornero received in 1982 the M.S. Degree in Systems and Control from the University of Manchester, Institute of Science and Technology and in 1985 the Ph.D. in Electrical Engineering at the Technical University of Valencia, Spain. He is currently Professor at the Department of Systems Engineering and Control, Head of the Robotics and Automation Research Group and also Director of the Design and Manufacture Institute of the Technical University of Valencia. He has been Visiting Professor at: the CIRSSE (NASA Center for Intelligent Robotics Systems for Space Exploration); the Rensselaer Polytechnic Institute at Troy (New York); and at the Department of Mechanical Engineering at the University of California (Berkeley). He is particularly interested in: modelling, control, and simulation of auto-guided-vehicles and robot arms; modelling, analysis, and control of multi-rate sampled data systems; as well as in collision detection/avoidance and automatic trajectory generation. Dr. Tornero has participated in many European research projects such as ESPRIT, BRITE, EUREKA and STRIDE, and in educational projects as ERAMUS, INTERCAMPUS, ALPHAS and TEMPUS. 